\title{
Troponin T elevation and long-term mortality after chronic obstructive pulmonary disease exacerbation
}

\author{
P.H. Brekke*, T. Omland*, S.H. Holmedal" , P. Smith* and V. Søyseth*
}

ABSTRACT: Patients with chronic obstructive pulmonary disease (COPD) are at increased risk of cardiovascular disease, exacerbations of which increase strain on the heart. The prognostic value of elevated circulating levels of cardiac Troponins seen during COPD exacerbations has been investigated.

From the Akershus hospital database, 897 patients discharged after treatment for COPD exacerbation in the period 2000-2003 were identified and followed-up until June 30, 2005. Median observation time was 1.9 yrs. In 396 patients, measurements of cardiac-specific troponin T (cTnT) were available. Levels of $\mathrm{cTnT} \geqslant 0.04 \mu \mathrm{g} \cdot \mathrm{L}^{-1}$ were considered elevated. Clinical data were retrieved from patient records and date of death was obtained from the Norwegian National Registry. In order to balance the nonrandomised nature of available cTnT measurements, an exposure propensity score (EPS) for cTnT sampling was calculated and used in regression analyses.

After adjusting for EPS in Cox regression analyses, elevated cTnT was significantly associated with increased all-cause mortality in the observation period, with a hazard ratio of 1.64 (95\% confidence interval 1.15-2.34).

In conclusion, chronic obstructive pulmonary disease patients with elevated cardiac-specific Troponin T during exacerbation are at increased risk of death after discharge.

KEYWORDS: Chronic obstructive pulmonary disease, cohort studies, prognosis, propensity score, troponin

hronic obstructive pulmonary disease (COPD) is a common and debilitating disease characterised by airflow limitation that is not fully reversible and usually progressive [1]. This disease represents an increasing health burden worldwide and is the only leading cause of death that still has a rising mortality rate in industrialised countries [2]. The most important risk factor in COPD is smoking, and the majority of patients are former or current smokers [1,3]. Thus, this patient population has an increased likelihood of developing other smoking-related diseases, including ischaemic heart disease (IHD) [4, 5]. In a recent review article on the role of comorbidities in COPD, SIN et al. [6] conclude that cardiovascular disease is a major cause of death in COPD. Although the underlying mechanisms are not fully understood, this could be due to chronic systemic and pulmonary inflammation.

Many COPD patients experience periods of disease exacerbation, with increased dyspnoea, sputum secretion, chest tightness and cough [7].
During exacerbations, bronchoconstriction and mucus production, alveolar hypoxia and resultant elevation of pulmonary vascular resistance can lead to hypoxaemia and increased cardiac frequency. Consequently, exacerbations represent an increased burden to the heart, and myocardial injury with release of cardiac-specific troponin $\mathrm{T}(\mathrm{cTnT})$ may occur.

Still, the literature on troponins in COPD exacerbations is sparse. Searching Medline and Embase databases, only one study on the prevalence of cTnT elevation in patients hospitalised for COPD exacerbation [8], and one study examining the inhospital prognostic value of cardiac troponin I (cTnI) elevation among COPD patients admitted to an intensive care unit (ICU) [8,9], were found. Accordingly, the objective of the current study was to explore the long-term prognostic value of elevated CTnT seen during COPD exacerbation.

\section{METHODS}

The study population consisted of an historic cohort of patients discharged, after treatment for

\section{AFFILIATIONS}

*Dept of Medicine and Faculty Division and

\#Dept of Radiology, Akershus University Hospital, Lørenskog, Norway.

CORRESPONDENCE

P.H. Brekke

Dept of Medicine Akershus University Hospital

N-1478 Lørenskog

Norway

Fax: 4767902125

E-mail: pal.brekke@ahus.no

Received:

February 092007

Accepted after revision:

October 302007

\section{SUPPORT STATEMENT}

This study received financial support from the Eva Zakariassen Memorial Fund.

\section{STATEMENT OF INTEREST}

Statements of interest for P.H. Brekke and T. Omland can be found at www.erj.ersjournals.com/misc/ statements.shtml 
COPD exacerbation, from Akershus University Hospital, Lørenskog, Norway, a 700-bed teaching hospital that serves suburban and countryside regions. Cases were identified using the hospital's patient database. Patients aged $\geqslant 40$ yrs who were admitted during the 4-year period January 1, 2000 and December 31, 2003 were discharged with a primary diagnosis of COPD exacerbation and had International Classification of Diseases (ICD) codes J44.0, J44.1 or COPD (ICD J44.X) as an underlying diagnosis combined with pneumonia (ICD J13J18.9) as the main diagnosis, were included. The choice of study period was based on data availability because changes in hospital database software made it harder to retrieve complete data from outside this time period. For patients with more than one admission during the inclusion period, the latest admission date was used. Patients were followed until death or June 30, 2005, whichever occurred first. Patients with previous diagnoses of sarcoidosis, interstitial lung disease or neuromuscular disease were excluded. In total, 897 patients met the inclusion criteria.

Mortality data were gathered from the Norwegian National Registry, which is based on a unique personal identification number for all Norwegian inhabitants.

Results of laboratory analyses performed within 24 hrs of hospital admission were retrieved from the hospital's laboratory database. If multiple results were available, average haemoglobin concentrations, maximum white blood cell (WBC) count, cTnT and C-reactive protein (CRP), and minimum creatinine levels were used. CRP levels were measured using an immunoturbidimetric method (Cobas Integra ${ }_{\circledR}$; Roche Diagnostics $\mathrm{GmbH}$, Mannheim, Germany), and the cTnT assay used by the hospital laboratory was Elecsys ${ }^{\circledR}$ Troponin T STAT (Roche Diagnostics $\mathrm{GmbH}$ ). The laboratory reported cTnT levels as measureable if $\geqslant 0.01 \mu \mathrm{g} \cdot \mathrm{L}^{-1}$, and elevated if $\geqslant 0.04 \mu \mathrm{g} \cdot \mathrm{L}^{-1}$, at which point the assay has $<10 \%$ coefficient of variation.

Patient data from emergency room presentation, including oxygenation measured by pulse oximetry, supplemental oxygen flow, arterial blood gas analysis (partial pressure of oxygen, partial pressure of carbon dioxide $\left.\left(\mathrm{PCO}_{2}\right), \mathrm{pH}\right)$, blood pressure, cardiac frequency, heart rhythm and respiratory frequency, in addition to medication use on admission and at discharge, were manually gathered from hospital records.

All diagnostic discharge ICD codes entered for each patient since 1987, until, but not including, the date of admission, were obtained from the hospital database. For each patient, groups of codes were used to construct a history of lung cancer, other cancers, diabetes, hypertension, IHD, congestive heart failure, atrial fibrillation, cerebrovascular disease, peripheral vascular disease and venous thromboembolism.

Patients were considered to have established IHD if there existed a previous diagnosis of myocardial infarction, hospitalisation for unstable angina or they had undergone percutaneous coronary intervention or coronary artery bypass surgery.

Spirometry data, if performed in a stable state $\geqslant 1$ week before hospital admission or 4 weeks after discharge, were gathered from patient records. Forced vital capacity (FVC), forced expiratory volume in one second (FEV1), height and weight were recorded, and body mass index (BMI), defined as the weight in kilograms divided by the square of height in metres, was calculated. FVC and FEV1 values are expressed as \% predicted using the European Community for Steel and Coal reference equations [10].

Available chest radiographs from the date of admission were re-examined in cooperation by V. Søyseth and S. H. Holmedal, who were blinded regarding clinical data. The following parameters were evaluated: cardiomegaly, hyperinflation, pneumonic infiltrates and pulmonary congestion.

The study was approved by the Data Inspectorate, and reviewed by the Regional Committee for Research Ethics.

\section{Statistical analyses}

All-cause mortality was used as the outcome measure. Continuous covariates were dichotomised: creatinine, cardiac frequency and oxygen saturation at the 10th and 90th percentiles; $\mathrm{PCO}_{2}, \mathrm{FEV} 1 \%$ pred and $\mathrm{BMI}$ at the 25th and 75th percentiles; and WBC count and CRP at the median. The concentration of cTnT was stratified at 0.01 and $0.04 \mu \mathrm{g} \cdot \mathrm{L}^{-1}$ for regression analyses.

First, baseline differences between patients with and without cTnT measurements were compared using univariate methods (unpaired t-test or Chi-squared as appropriate).

Secondly, variables with a p-value $\leqslant 0.15$ in the univariate analyses and/or deemed to be potentially associated with the decision to measure cTnT, were entered into a logistic regression analysis model. This model was then used to calculate individual exposure propensity scores (EPS) for the probability of cTnT measurement [11].

Thirdly, age- and sex-adjusted relative mortality was assessed using Mantel-Haenszel test for incidence density data.

Fourthly, all covariates with a significance level $\leqslant 0.15$ in the stratified analyses were entered into a Cox proportional hazards model for all-cause mortality. Models for the subgroup with cTnT measurement and for the full cohort were compared, using EPS as a balancing score.

Finally, the same Cox models were applied to more restricted cohorts, excluding patients with lung cancer, IHD and neversmokers.

Time since discharge was used as the underlying time variable. The proportional hazard assumption was tested using traditional log-log plots and Schoenfeld's residuals on partial likelihood [12]. Results are reported as odds ratio (OR) for logistic regression analysis models, and hazard ratio (HR) for Cox analyses, with $95 \%$ confidence intervals (CIs) in parentheses.

\section{RESULTS}

The mean age of the cohort at discharge was 70.9 yrs and $50.8 \%$ were female. Only $55(6 \%)$ of the patients had never smoked. Spirometry data was available for 697 patients. Of these, $608(87 \%)$ had an FEV1/FVC ratio $\leqslant 0.7$. The level of cTnT had been measured in 396 patients. The median time of observation was 1.9 yrs. After discharge, cumulative survival was 75.6 and $65.2 \%$ at 1 and 2 yrs, respectively. 

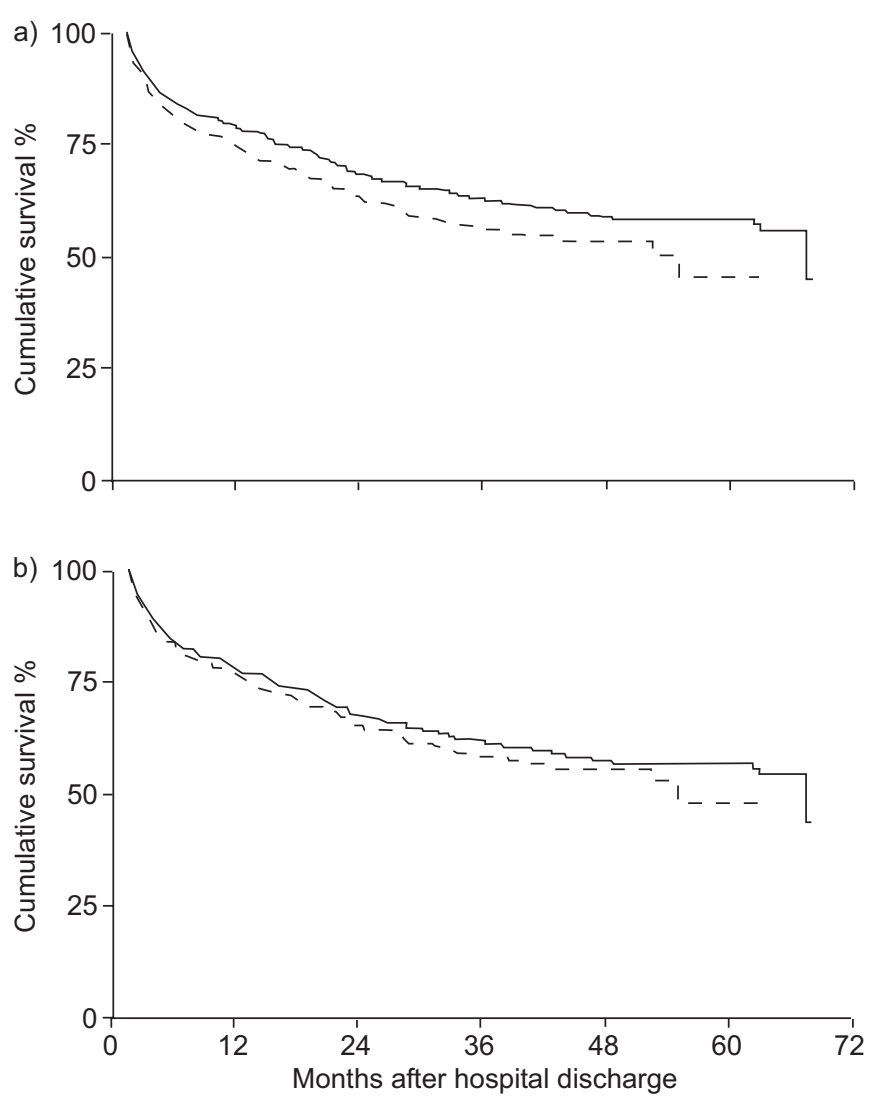

FIGURE 1. a) Unadjusted and b) exposure propensity score-adjusted KaplanMeier survival curves for measured (----) versus not measured (-) cardiac-specific troponin T levels.

In univariate analysis, there was a slight difference in survival (HR 1.25 (1.01-1.54); $\mathrm{p}=0.04$, Cox regression) between patients who had had cTnT measured and those who did not (fig. 1a). However, adjusting for EPS, which assigns a probability of sampling to each patient, the mortality risk was nearly equalised and statistically nonsignificant (HR 1.12 (0.90-1.39); $p=0.33$, Cox regression; fig. $1 b)$. There was a highly significant difference in overall survival $(p<0.0001$, age-stratified $\log$ rank) in patients with versus without cTnT elevation, and an equally significant trend for mortality as a function of cTnT concentration when stratified (fig. 2).

Baseline characteristics for the groups with and without cTnT measurements are presented in table 1 . The covariates associated with cTnT measurement for the full cohort, and thus included in the calculation of the EPS, are presented with ORs for measurement. Patients with reduced FEV1 or hyperinflation on radiograph had a lower probability of cTnT sampling, whereas a history of IHD, use of aspirin, or chest radiograph signs of pulmonary congestion increased the odds of cTnT measurement.

In 173 of the 396 samples, cTnT was at a measureable level with a median concentration of $0.04 \mu \mathrm{g} \cdot \mathrm{L}^{-1}$ and 95 th percentile of $0.28 \mu \mathrm{g} \cdot \mathrm{L}^{-1}$. Patients with elevated $\mathrm{cTnT}$ presented with higher WBC counts and creatinine levels, and had slightly worse pulmonary function. Cardiomegaly was more common

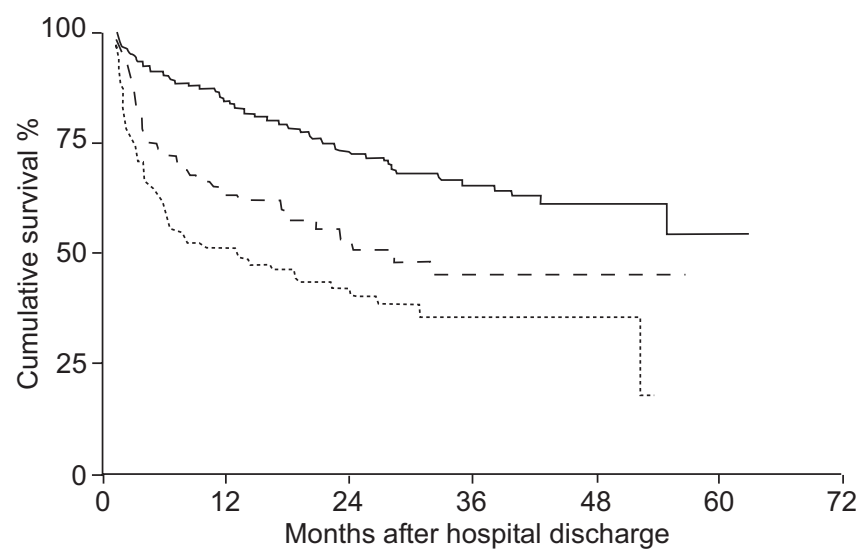

FIGURE 2. Kaplan-Meier survival curve for cardiac-specific troponin $T$ concentration strata: $<0.01 \mu \mathrm{g} \cdot \mathrm{L}^{-1}(-) ; 0.01-0.03 \mu \mathrm{g} \cdot \mathrm{L}^{-1}(---)$; and $\geqslant 0.04 \mu \mathrm{g} \cdot \mathrm{L}^{-1}$ $(\cdots \cdot)$.

among patients with elevated $c \operatorname{TnT}$, whereas infiltrates were more often seen in patients without cTnT elevation.

Hypertension, low $\mathrm{pH}$, elevated CRP, signs of infiltrate or hyperinflation on chest radiography, and use of $\beta$-adrenergic blockers, angiotensin-converting enzyme inhibitors, warfarin or aspirin were covariates initially tested in stratified analyses, which did not have an association with mortality $(p<0.15)$ and thus were not included in the regression models.

Table 2 shows two multivariate Cox-survival models: one for the subgroup of patients with cTnT measurement and one for the entire cohort. The same variables were used in both models, with the addition of EPS as a balancing score in the full cohort model in order to adjust for the potential bias in the selection of patients.

In the regression analyses, several covariates had a significant positive association with mortality; cTnT elevation, old age, anaemia, reduced FEV1, low BMI, low oxygen saturation, cardiomegaly and a history of IHD, thromboembolism or cancer were among the major contributors, whereas neversmokers and statin users had a reduced mortality risk. Removing EPS from the full cohort model resulted in only a modest change in the HR $(95 \% \mathrm{CI})$ for $\mathrm{cTnT} \geqslant 0.04 \mu \mathrm{g} \cdot \mathrm{L}^{-1}$ to $1.61(1.13-2.29)$.

Since cTnT concentration was highly skewed, a log-transformed variable was generated and substituted for the stratified cTnT variable used in the models in table 2. The association with mortality was highly significant, with an HR per SD for $\log (\mathrm{cTnT})$ of 1.24 (1.04-1.47) for the $\mathrm{cTnT}$ subgroup.

Patients with IHD, lung cancer and never-smokers were then sequentially excluded from the models in table 2 . The adjusted HRs for cTnT are presented in table 3. In all the reduced models, elevated cTnT remained significantly associated with an increased mortality risk for the full cohort, but only marginally significant in the subgroup model. The HRs did not change appreciably between any of the smaller models.

Six patients received a secondary diagnosis of acute myocardial infarction during the index admission. Excluding these 


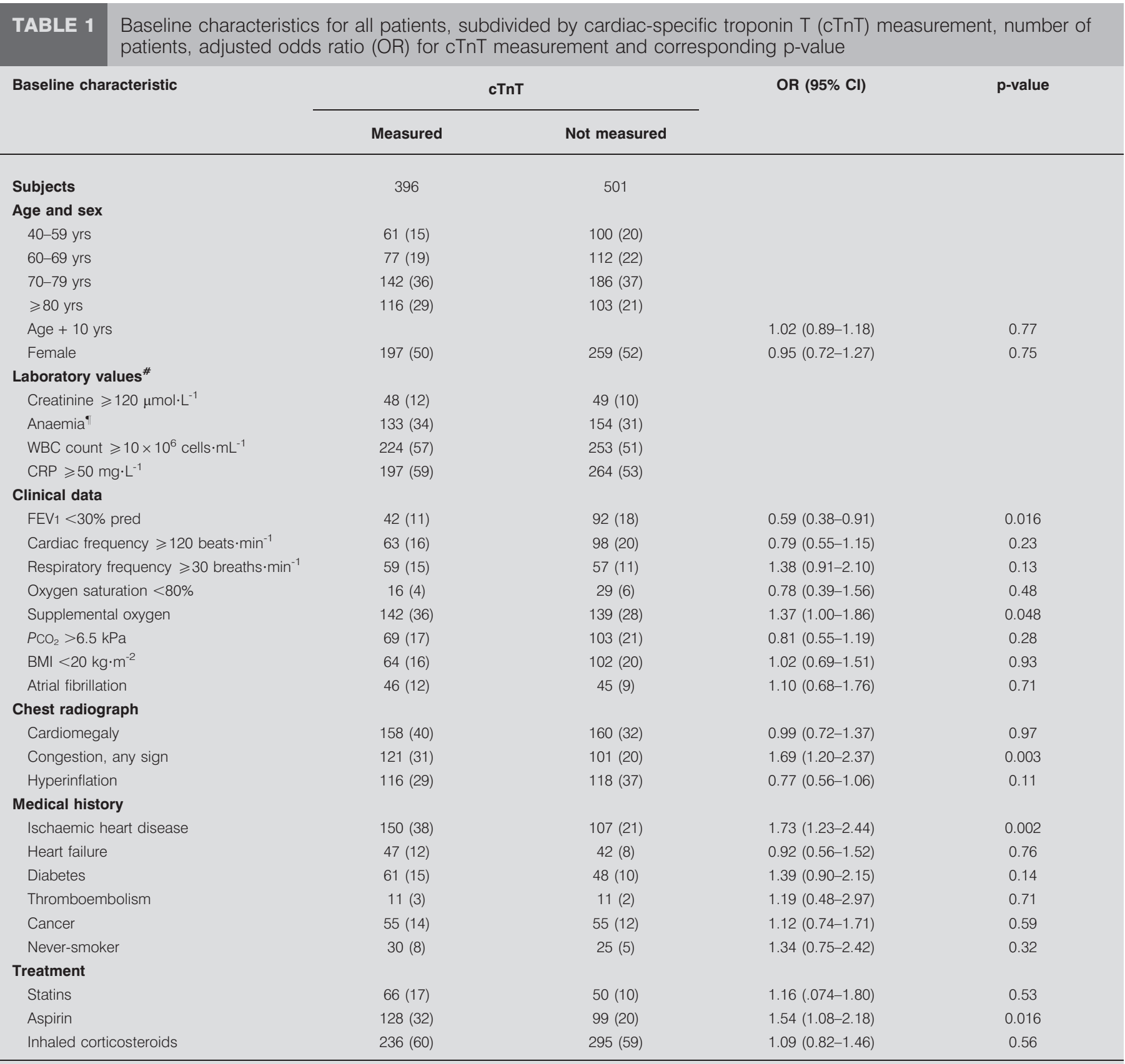

Data are presented as $n(\%)$, unless otherwise stated. Cl: confidence interval; WBC: white blood cell; CRP: C-reactive protein; FEV1: forced expiratory volume in one

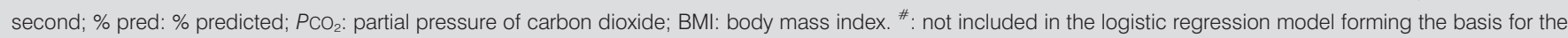
exposure propensity score calculation; ": haemoglobin concentration $<12 \mathrm{mg} \cdot \mathrm{dL}^{-1}$ for females and $<13 \mathrm{mg} \cdot \mathrm{dL}^{-1}$ for males.

patients from the analysis did not influence the results. None of the models violated the proportional hazard assumption.

\section{DISCUSSION}

The present authors found that elevated cTnT seen during hospitalisation for COPD exacerbation was a strong and independent prognostic factor for mortality after discharge, even after adjusting for multiple well-known determinants of mortality. To the best of the present authors' knowledge this association between troponin elevation and mortality in patients discharged from hospital after a COPD exacerbation is a novel finding. Moreover, it may be important for risk stratification and treatment of patients hospitalised for COPD exacerbation.

One obvious limitation of the present study is the selection of patients from whom cTnT was sampled. Of the 897 COPD patients in the initial cohort, the physicians on call decided to analyse cTnT in 396. According to hospital guidelines at the time of the study, troponin $\mathrm{T}$ testing was recommended in all patients with chest pain and clinical suspicion of acute 
TABLE 2 Hazard ratio (HR) from multivariate Cox regression for the subgroup with cardiac-specific troponin T (cTnT) measurement and the full cohort according to characteristic

\begin{tabular}{|c|c|c|c|c|}
\hline & \multicolumn{2}{|c|}{ cTnT measured $^{\#}$} & \multicolumn{2}{|c|}{ All patients } \\
\hline & $\mathrm{HR}^{+}(95 \% \mathrm{Cl})$ & $\mathrm{p}$-value & $\mathrm{HR}^{+}(95 \% \mathrm{Cl})$ & $\mathrm{p}$-value \\
\hline \multicolumn{5}{|l|}{ Age and sex } \\
\hline Age $+10 \mathrm{yrs}$ & $1.57(1.29-1.92)$ & $<0.001$ & $1.64(1.44-1.86)$ & $<0.001$ \\
\hline Female & $0.82(0.57-1.19)$ & 0.30 & $0.83(0.66-1.04)$ & 0.11 \\
\hline Not measured & & & 1 & \\
\hline$<0.01 \mu \mathrm{g} \cdot \mathrm{L}^{-1}$ & 1 & & $1.02(0.78-1.35)$ & 0.86 \\
\hline $0.01-0.03 \mu \mathrm{g} \cdot \mathrm{L}^{-1}$ & $1.23(0.78-1.93)$ & 0.38 & $1.16(0.79-1.71)$ & 0.45 \\
\hline$\geqslant 0.04 \mu \mathrm{g} \cdot \mathrm{L}^{-1}$ & $1.66(1.07-2.56)$ & 0.023 & $1.64(1.15-2.34)$ & 0.006 \\
\hline Creatinine $\geqslant 120 \mu \mathrm{mol} \cdot \mathrm{L}^{-1}$ & $1.48(0.95-2.32)$ & 0.08 & $1.25(0.91-1.71)$ & 0.17 \\
\hline Anaemia $^{\S}$ & $1.29(0.91-1.84)$ & 0.15 & $1.29(1.02-1.63)$ & 0.035 \\
\hline Oxygen saturation $<80 \%$ & $2.13(1.03-4.41)$ & 0.042 & $2.61(1.71-3.98)$ & $<0.001$ \\
\hline Supplemental oxygen & $1.34(0.95-1.87)$ & 0.09 & $1.42(1.09-1.85)$ & 0.009 \\
\hline $\mathrm{PCO}_{2}>6.5 \mathrm{kPa}$ & $1.22(0.78-1.93)$ & 0.39 & $1.31(0.98-1.75)$ & 0.07 \\
\hline $\mathrm{BMl}<20 \mathrm{~kg} \cdot \mathrm{m}^{-2}$ & $1.72(1.13-2.63)$ & 0.012 & $1.48(1.13-1.95)$ & 0.005 \\
\hline Atrial fibrillation & $0.98(0.60-1.59)$ & 0.92 & $1.4(1.03-1.90)$ & 0.03 \\
\hline \multicolumn{5}{|l|}{ Chest radiograph } \\
\hline Cardiomegaly & $1.72(1.18-2.49)$ & 0.005 & $1.42(1.11-1.83)$ & 0.005 \\
\hline Congestion, any sign & $0.7(0.48-1.01)$ & 0.06 & $0.79(0.57-1.10)$ & 0.16 \\
\hline \multicolumn{5}{|l|}{ Medical history } \\
\hline Ischaemic heart disease & $1.36(0.96-1.94)$ & 0.08 & $1.54(1.05-2.26)$ & 0.026 \\
\hline Heart failure & $1.38(0.87-2.18)$ & 0.17 & $1.26(0.91-1.75)$ & 0.16 \\
\hline Inhaled corticosteroids & $0.9(0.64-1.26)$ & 0.53 & $0.87(0.69-1.09)$ & 0.22 \\
\hline EPS & & & $0.5(0.07-3.53)$ & 0.48 \\
\hline
\end{tabular}

$\mathrm{Cl}$ : confidence interval; WBC: white blood cell; FEV1: forced expiratory volume in one second; \% pred: \% predicted; $\mathrm{PCO}_{2}$ : partial pressure of carbon dioxide; $\mathrm{BMI}$ : body mass index; EPS: exposure propensity score. ${ }^{\#}: \mathrm{n}=396 ;{ }^{\bullet}: \mathrm{n}=897 ;^{+}$: adjusted for all other variables in the table; ${ }^{\text {s. }}$, haemoglobin concentration $<12 \mathrm{mg} \cdot \mathrm{dL}^{-1}$ for females and $<13 \mathrm{mg} \cdot \mathrm{dL}^{-1}$ for males.

coronary syndromes. In addition, troponin $\mathrm{T}$ testing was mentioned as an option in patients with clinical suspicion of myocarditis, acute heart failure and pulmonary embolism. The nonrandomised selection may introduce a bias in the effect estimate, even though the unadjusted comparison of survival of patients with CTnT measurements with those without, showed only a very small difference in survival between the two groups. However, by adjusting for the propensity score EPS, which assigns a probability of cTnT sampling to all 897 patients in the cohort, the survival difference is almost completely equalised and statistically nonsignificant. Propensity scores have been shown to remove or considerably reduce bias in background covariates, albeit at the cost of reduced statistical power [11, 13]. Adding or removing EPS from the final Cox model caused only marginal changes in the HR estimates for cTnT. Thus, the selection of patients for cTnT measurement did not influence the association between cTnT and mortality among these patients. Still, the conclusions drawn from the present study should be considered as hypothesis generating.

Excluding subgroups of patients with lung cancer, established IHD or never-smokers from the analysis minimally affected the HR estimates for cTnT elevation in any model. cTnT remained a significant predictor of mortality risk in the full cohort models but caused the association to be only borderline 


\begin{tabular}{|c|c|c|c|c|}
\hline \multirow{2}{*}{ TABLE 3} & \multicolumn{2}{|c|}{ cTnT measured } & \multicolumn{2}{|c|}{ All patients } \\
\hline & HR $(95 \%$ Cl) & $\mathrm{p}$-value & HR $(95 \% \mathrm{Cl})$ & p-value \\
\hline \multicolumn{5}{|c|}{ Never-smokers excluded } \\
\hline$<0.01 \mu \mathrm{g} \cdot \mathrm{L}^{-1}$ & 1 & & $1.07(0.81-1.41)$ & 0.64 \\
\hline $0.01-0.03 \mu \mathrm{g} \cdot \mathrm{L}^{-1}$ & $1.07(0.66-1.75)$ & 0.77 & $1.07(0.71-1.62)$ & 0.74 \\
\hline$\geqslant 0.04 \mu \mathrm{g} \cdot \mathrm{L}^{-1}$ & $1.54(0.97-2.44)$ & 0.07 & $1.62(1.12-2.34)$ & 0.01 \\
\hline \multicolumn{5}{|c|}{ Ischaemic heart disease excluded } \\
\hline \multicolumn{5}{|l|}{ cTnT } \\
\hline \multicolumn{5}{|c|}{ Lung cancers excluded } \\
\hline \multicolumn{5}{|l|}{ cTnT } \\
\hline Not measured & & & 1 & \\
\hline$<0.01 \mu \mathrm{g} \cdot \mathrm{L}^{-1}$ & 1 & & $1.05(0.79-1.40)$ & 0.75 \\
\hline $0.01-0.03 \mu \mathrm{g} \cdot \mathrm{L}^{-1}$ & $1.28(0.78-2.10)$ & 0.32 & $1.2(0.79-1.81)$ & 0.40 \\
\hline$\geqslant 0.04 \mu \mathrm{g} \cdot \mathrm{L}^{-1}$ & $1.54(0.96-2.48)$ & 0.07 & $1.56(1.06-2.29)$ & 0.023 \\
\hline
\end{tabular}

HR: hazard ratio; $\mathrm{Cl}$ : confidence interval.

significant in the cTnT subgroup models. This effect can be ascribed partly to loss of statistical power and partly to patient selection.

As to other markers of myocardial injury, the present authors' hospital laboratory did not offer cTnI analysis in the time period of the study, but cTnI is likely to have yielded similar results. The inferior signal to noise ratio of $\mathrm{MB}$ isoform of creatine kinase (CK-MB) analyses may have resulted in reduced ability to identify patients at increased risk. Of the very few patients for whom CK-MB was sampled, all of them had had cTnT measured as well.

Using the latest admission date was an arbitrary decision made during data gathering. Since the choice of inclusion date could influence neither the population sample nor the number of deaths, the present authors believe the effect on the results is marginal.

Long-term oxygen therapy (LTOT) has been shown to improve survival in select groups of COPD patients with hypoxaemia. Since hypoxic patients may be more vulnerable to myocardial injury, adherence to guidelines for LTOT could influence the association between cTnT and mortality. While the present authors believe that recommendations for LTOT were adhered to during the study period, the exact data on LTOT in the present cohort was not available.

There are several alternative explanations for cTnT elevation which should be considered in the present patient population. First, physicians caring for the patients may have overlooked other conditions that are known to cause troponin elevation, such as pulmonary embolism, renal failure or heart failure [14, 15].
Pulmonary embolism is not infrequent as a cause of dyspnoea in COPD patients [16] but the diagnosis remains elusive in clinical practice. History of thromboembolic disease was included in the present analyses in an effort to control for embolism as a cofactor. Furthermore, serum creatinine was included to adjust for renal function. The study by BAILLARD et al. [9] reporting increased in-hospital mortality in COPD exacerbations requiring ICU treatment has been criticised for not taking markers of acute heart failure into account [17]. The present authors' hospital laboratory did not provide analysis of B-type natriuretic peptide at the time of the study. In order to account for, and provide an index of, acute pulmonary congestion, chest radiographs were re-evaluated. Information on cephalisation obtained from chest radiographs has been shown to correlate strongly with acute congestive heart failure severity [18].

Secondly, a COPD exacerbation could in itself cause sufficient strain on the heart to induce myocardial cell necrosis. Oedema, mucus hypersecretion and bronchoconstriction may cause further ventilation impairment, and alveolar hypoxia may cause constriction of pulmonary arterioles and increased pulmonary artery pressure, disturbing perfusion. Additionally, respiratory muscle fatigue and abnormal respiratory patterns further deteriorate arterial blood gases. Tachycardia, hypoxaemia and dilatation of the right ventricle often seen in COPD exacerbations, and complications such as pulmonary arterial hypertension, are all factors that may cause troponin release [14, 19].

Thirdly, there is increasing awareness of IHD as a major contributor to morbidity and mortality in the present patient population [5, 6, 20,21]. The majority of patients are current or former smokers, and are at increased risk of developing other 
smoking-related diseases. While troponin release is seen in COPD even in patients with normal coronary arteries on angiography [15], this does not preclude the possibility that many COPD patients actually have underlying quiescent IHD. If the coronary circulation is already impaired, the extra strain put on the heart by a COPD exacerbation could lead to an oxygen supply-demand mismatch, causing myocardial damage.

Finally, the increased inflammatory response in COPD exacerbations [3, 22] may amplify the inflammatory processes associated with atherosclerosis and atherothrombosis [6, 23]. The present authors have previously published a paper suggesting a survival benefit of statin treatment in COPD [24], an effect which may be linked to the pleiotropic antiinflammatory effect of statins.

The diagnosis of COPD in the present study was performed at discharge, based on all available clinical data. Frequently, deciding whether or not COPD exacerbations are accompanied by pneumonia is difficult. Thus, patients coded with pneumonia as the main diagnosis and COPD as the underlying diagnosis were included. A physician specialised in internal medicine or pulmonary medicine verified the diagnoses. While the diagnosis was not based on specific criteria, such that as suggested by the Global Initiative for Chronic Obstructive Lung Disease (GOLD) [1], the study population was well defined and representative of COPD patients seen in pulmonary care units in Western countries.

The proper and correct coding of causes of death in COPD is difficult, and death certificates may both under- and overestimate COPD and comorbidities as underlying causes of death, leading to serious misclassification [6, 20]. Thus, all-cause mortality was chosen as the outcome measure. The overall mortality rate in the present study was comparable with the long-term mortality rate in previous studies of patients hospitalised with COPD exacerbation [25, 26]. Thanks to the unique personal identification number assigned to all Norwegian inhabitants, linkage between registries was possible allowing mortality data to be gathered from the Norwegian National Registry. This ensured complete follow-up of the cohort and made misclassification of the outcome highly unlikely.

In conclusion, assessment of cardiac-specific troponin $\mathrm{T}$ levels in patients with chronic obstructive pulmonary disease exacerbation on hospital admission permits identification of patients at increased risk of later death. Considering the dearth of chronic obstructive pulmonary disease treatments that actually have an impact on prognosis, and the likelihood that at least some of the patients with elevated troponin levels have treatable but undiagnosed comorbidities, the present findings provide an impetus to further characterise the pathophysiological correlates and determinants of troponin elevation in chronic obstructive pulmonary disease.

\section{ACKNOWLEDGEMENTS}

The authors wish to thank F. Mahmood and L.M.D. Søyseth for their contribution in collecting data from the medical records.

\section{REFERENCES}

1 Pauwels RA, Buist AS, Calverley PM, Jenkins CR, Hurd SS, GOLD Scientific Committee. Global strategy for the diagnosis, management, and prevention of chronic obstructive pulmonary disease. NHLBI/WHO Global Initiative for Chronic Obstructive Lung Disease (GOLD) Workshop summary. Am J Respir Crit Care Med 2001; 163: 1256-1276.

2 Murray CJ, Lopez AD. Alternative projections of mortality and disability by cause 1990-2020: Global Burden of Disease Study. Lancet 1997; 349: 1498-1504.

3 Barnes PJ. Chronic obstructive fulmonary disease. N Engl J Med 2000; 343: 269-280.

4 Hole DJ, Watt GC, Davey-Smith G, Hart CL, Gillis CR, Hawthorne VM. Impaired lung function and mortality risk in men and women: findings from the Renfrew and Paisley prospective population study. BMJ 1996; 313: 711-715.

5 Vollset SE, Tverdal A, Gjessing HK. Smoking and deaths between 40 and 70 years of age in women and men. Ann Intern Med 2006; 144: 381-389.

6 Sin DD, Anthonisen NR, Soriano JB, Agusti AG. Mortality in COPD: role of comorbidities. Eur Respir J 2006; 28: 1245-1257.

7 Currie GP, Wedzicha JA. ABC of chronic obstructive pulmonary disease. Acute exacerbations. BMJ 2006; 333: 87-89.

8 Harvey MG, Hancox RJ. Elevation of cardiac troponins in exacerbation of chronic obstructive pulmonary disease. Emerg Med Australas 2004; 16: 212-215.

9 Baillard C, Boussarsar M, Fosse JP, et al. Cardiac troponin I in patients with severe exacerbation of chronic obstructive pulmonary disease. Intensive Care Med 2003; 29: 584-589.

10 Quanjer PH, Tammeling GJ, Cotes JE, Pedersen OF, Peslin R, Yernault JC. Lung volumes and forced ventilatory flows. Report Working Party Standardization of LungFunction Tests European-Community for Steel and Coal. Official Statement of the European Respiratory Society. Eur Respir J 1993; 6: Suppl. 16, 5-40.

11 D'Agostino RB Jr. Propensity score methods for bias reduction in the comparison of a treatment to a nonrandomized control group. Stat Med 1998; 17: 2265-2281.

12 Marubini E, Valsecchi. Validation of the proportional hazard models. In: Marubini E, Valsecchi MG. Analysing Survival Data from Clinical Trials and Observational Studies. West Sussex, John Wiley \& Sons Ltd, 1995; pp. 251-254.

13 Stürmer T, Schneeweiss S, Brookhart MA, Rothman KJ, Avorn J, Glynn RJ. Analytic strategies to adjust confounding using exposure propensity scores and disease risk scores: nonsteroidal antiinflammatory drugs and shortterm mortality in the elderly. Am J Epidemiol 2005; 161: 891-898.

14 Korff S, Katus HA, Giannitsis E. Differential diagnosis of elevated troponins. Heart 2006; 92: 987-993.

15 Mahajan N, Mehta Y, Rose M, Shani J, Lichstein E. Elevated troponin level is not synonymous with myocardial infarction. Int J Cardiol 2006; 111: 442-449.

16 Tillie-Leblond I, Marquette CH, Perez T, et al. Pulmonary embolism in patients with unexplained exacerbation of chronic obstructive pulmonary disease: prevalence and risk factors. Ann Intern Med 2006; 144: 390-396.

17 Joffe A. Comments on elevated cardiac troponin in patients with COPD. Intensive Care Med 2004; 30: 732. 
18 Knudsen CW, Omland T, Clopton P, et al. Diagnostic value of B-Type natriuretic peptide and chest radiographic findings in patients with acute dyspnea. Am J Med 2004; 116: 363-368.

19 Torbicki A, Kurzyna M, Kuca P, et al. Detectable serum cardiac troponin $\mathrm{T}$ as a marker of poor prognosis among patients with chronic precapillary pulmonary hypertension. Circulation 2003; 108: 844-848.

20 Hansell AL, Walk JA, Soriano JB. What do chronic obstructive pulmonary disease patients die from? A multiple cause coding analysis. Eur Respir J 2003; 22: 809-814.

21 Sin DD, Man SFP. Why are patients with chronic obstructive pulmonary disease at increased risk of cardiovascular diseases? The potential role of systemic inflammation in chronic obstructive pulmonary disease. Circulation 2003; 107: 1514-1519.

22 Wouters EFM. Local and systemic inflammation in chronic obstructive pulmonary disease. Proc Am Thorac Soc 2005; 2: 26-33.

23 Hansson GK. Inflammation, atherosclerosis, and coronary artery disease. N Engl J Med 2005; 352: 1685-1695.

24 Søyseth V, Brekke PH, Smith P, Omland T. Statin use is associated with reduced mortality in COPD. Eur Respir J 2007; 29: 279-283.

25 Almagro P, Calbo E, Ochoa de Echaguen A, et al. Mortality after hospitalization for COPD. Chest 2002; 121: 1441-1448.

26 Groenewegen KH, Schols AM, Wouters EF. Mortality and mortality-related factors after hospitalization for acute exacerbation of COPD. Chest 2003; 124: 459-467. 